\title{
SOME TAUBERIAN THEOREMS FOR EULER AND BOREL SUMMABILITY
}

\author{
J. A. FRIDY \\ Department of Mathematics \\ Kent State University \\ Kent, Ohio $44252^{\circ}$ U.S.A. \\ K. L. ROBERTS \\ Department of Mathematics \\ The University of Western Ontario \\ London, Ontario \\ Canada
}

(Received August 6, 1979 and in revised form December 7, 1979)

ABSTRACT. The well-known summability methods of Euler and Borel are studied as mappings from $\ell^{1}$ into $\ell^{1}$. In this $\ell-\ell$ setting, the following Tauberian results are proved: if $\mathrm{x}$ is a sequence that is mapped into $\ell^{1}$ by the Euler-Knopp method $\mathrm{E}_{\mathrm{r}}$ with $r>0$ ( or the Borel matrix method) and $x$ satisfies $\sum_{n=0}^{\infty}\left|x_{n}-x_{n+1}\right| \sqrt{n}^{n}<\infty$, then $x$ itself is in $\ell^{1}$.

KEY WORDS AND PHRASES. Tauberian condition, $\ell-\ell$ method, Euler-Knopp means, Borel exponential method.

1980 MATHEMATICS SUBJECT CLASSIFICATION CODES: Primary - 40E05, 40G05, 40G10; Secondary - 40025 . 


\section{INTRODUCTION.}

In $[2$, p. 121], G. H. Hardy described a Tauberian theorem as one which asserts that a particular summability method cannot sum a divergent series that oscillates too slowly. In this paper we shall state the results in sequence-tosequence form, so a typical order-type Tauberian theorem for a method A would have the form, "if $x$ is a sequence such that $A x$ is convergent and $\Delta x_{k}=x_{k}-x_{k+1}=0\left(d_{k}\right)$, then $x$ itself is convergent." Our present task is not to give more theorems in the setting of ordinary convergence, but rather, we shall develop analogous results for methods that map $\ell^{1}$ into $\ell^{1}$. Such a transformation is called an $l-l$ method, and we shall henceforth write $l$ for $l^{1}$. In [5] Knopp and Lorentz proved that the matrix A determines an $l-l$ method if and only if $\sup _{k} \sum_{n=0}^{\infty}\left|a_{n k}\right|<\infty$. In order to prove Tauberian theorems in an $l-l$ setting, it is necessary to formulate an $l-l$ analogue of the above Tauberian condition $\Delta x_{k}=o\left(d_{k}\right)$. Since this condition means that $\Delta x / d$ is in $c_{0}$, an $l-l$ analogue would be " $\Delta x / d$ is in $l$," which we sha11 write in series form as $\sum_{k=0}^{\infty}\left|\Delta x_{k}\right| / d_{k}<\infty$.

\section{EULER-KNOPP AND BOREL $\ell-\ell$ METHODS.}

The Euler-Knopp means [6, pp. 56-60] are given by the matrix

$$
E_{r}[n, k]= \begin{cases}\left(\begin{array}{l}
n \\
k
\end{array}\right) r^{k}(1-r)^{n-k}, & \text { if } k \leq n, \\
0, & \text { if } k>n .\end{cases}
$$

In [1, Theorem 4] it is shown that $E_{r}$ determines an $l-l$ method if and only if $0<\mathrm{r} \leq 1$. Moreover, for such $\mathrm{r}, \mathrm{E}_{\mathrm{r}}^{-1}[l] \neq l$.

The customary form of Borel exponential summability is the sequence-tofunction transformation ([2, p. 182], [6, p. 54]) given by

$$
\text { if } \lim _{t-\infty}\left\{e^{-t} \sum_{k=0}^{\infty} x k^{t} / k !\right\}=L \text {, then } x \text { is Borel summable to } L \text {. }
$$

In order to consider this method in an $\ell-l$ setting, we must modify it into a 
sequence-to-sequence transformation. This can be achieved by letting tend to $\infty$ through integer values and considering the resulting sequence Bx. Then $B$ is the Borel matrix method $[6$, p. 56], which is given by the matrix

$$
b_{n k}=e^{-n_{n} k / k !}
$$

By a direct application of the Knopp-Lorentz Theorem [5], one can show that B is an $\ell-l$ matrix. We sha11 not use this direct approach, however, because the assertion will follow from our first theorem, which is an inclusion theorem between $B$ and $\mathrm{E}_{\mathbf{r}}$.

THEOREM 1. If $r>0$ and $x$ is a sequence such that $E_{r} x$ is in $l$, then $B x$ is in $\ell$.

PROOF. We use the familiar technique of showing that $\mathrm{BE}_{\mathrm{r}}^{-1}$ is an $l-l$ matrix. Since $B x=\left(B E_{r}^{-1}\right) E_{r} x$, this will ensure that $B x$ is in $l$ whenever $E_{r} x$ is in $l$. Since $E_{r}^{-1}=E_{1 / r}$, we replace $1 / r$ by $s$ and show that $B E_{s}$ is an $l-l$ matrix for all positive s. The $n, k$-th term of $\mathrm{BE}_{s}$ is given by

$$
\begin{aligned}
B_{s}[n, k] & =\sum_{j=k}^{\infty} \frac{e^{-n_{n} j}}{j !}\left(\begin{array}{l}
j \\
k
\end{array}\right)(1-s)^{j-k_{s} k} \\
& =\frac{e^{-n n_{s} k}}{k !} \sum_{j=k}^{\infty} \frac{n^{j-k}}{(j-k) !}(1-s)^{j-k} \\
& =\frac{(n s)^{k} e^{-n s}}{k !} .
\end{aligned}
$$

Summing the $k$-th column of $\mathrm{BE}_{s}$, we get

$$
\begin{aligned}
\sum_{n=0}^{\infty}\left|B E_{s}[n, k]\right| & =\frac{1}{k !} \sum_{n=0}^{\infty}(n s)^{k} e^{-n s} \\
& =0\left(\frac{1}{k !} \int_{0}^{\infty}(t s)^{k} e^{-t s} d t\right) \\
& =0(1 / s) .
\end{aligned}
$$


Hence, $\sup _{k} \sum_{n=0}^{\infty}\left|B E_{s}[n, k]\right|<\infty$, so $\mathrm{BE}_{s}$ is an $l-l$ matrix.

Combining Theorem 1 with the knowledge that $\mathrm{E}_{\mathrm{r}}$ is an $\ell-l$ matrix, we get the following result as an immediate corollary.

THEOREM 2. The Borel matrix B determines an $l-l$ method.

In addition to the inclusion relation given in Theorem 1, we can show that the $\ell-l$ method $B$ is strictly stronger than all $E_{r}$ methods by the following example.

EXAMPLE. Suppose $r>0$ and $x_{k}=(-s)^{k}$, where $s \geq-1+2 / r$; then $B x$ is in $l$ but $E_{r} X$ is not in $\ell$. For,

$$
(B x)_{n}=\Sigma_{k=0}^{\infty} e^{-n} \frac{n^{k}}{k !}(-s)^{k}=e^{-n} e^{-s n}=e^{-n(s+1)},
$$

and

$$
\left(E_{r} x\right)_{n}=\Sigma_{k=0}^{n}\left(\begin{array}{l}
n \\
k
\end{array}\right)(1-r)^{n-k}(-r s)^{k}=(1-r-r s)^{n} .
$$

By solving $-1<1-r^{\prime}-r s<1$, we see that $E_{r} x$ is in $l$ if and only if $-1<s<-1+2 / r$.

\section{TAUBERIAN THEOREMS.}

We are now ready to prove the principal results which show that $B$ and $E_{r}$ can not map a sequence from $\sim l$ into $l$ if the sequence oscillates too slowly.

THEOREM 3. If $x$ is a sequence such that $B x$ is in $l$ and

$$
\sum_{r=0}^{\infty}\left|\Delta x_{r}\right| \sqrt{r}<\infty
$$

then $x$ is in $l$.

PROOF. It suffices to show that $B X-x$ is in $\ell$; that is, $\Sigma_{n=0}^{\infty}\left|\Sigma_{k=0}^{\infty} b_{n k} x_{k}-x_{n}\right|<\infty$. Since $\Sigma_{k=0}^{\infty} b_{n k}=1$ for each $n$, this sum can be written as $\Sigma_{n=0}^{\infty}\left|\Sigma_{k=0}^{\infty} b_{n k}\left(x_{k}-x_{n}\right)\right|$, and so it suffices to show that $A=\Sigma_{n=0}^{\infty} \Sigma_{k=0}^{\infty} b_{n k} \mid x_{k}-x_{n} k^{\infty}$. 
We can write $A=C+D$, where

$$
C=\sum_{n=0}^{\infty} \Sigma_{k=0}^{\infty} b_{n k}\left|x_{k}-x_{n}\right|
$$

and

$$
D=\sum_{n=0}^{\infty} \Sigma_{k=n+1}^{\infty} b_{n k}\left|x_{k}-x_{n}\right|
$$

Then

$$
\begin{aligned}
C & \leq \Sigma_{n=0}^{\infty} \Sigma_{k=0}^{n-1} b_{n k} \Sigma_{r=k}^{n-1}\left|\Delta x_{r}\right| \\
& =\Sigma_{r=0}^{\infty}\left|\Delta x_{r}\right| \Sigma_{n=r+1}^{\infty} \Sigma_{k=0}^{r} b_{n k} \\
& =\Sigma_{r=0}^{\infty}\left|\Delta x_{r}\right| C_{r} \text {, say. }
\end{aligned}
$$

A1so,

$$
\begin{aligned}
\mathrm{D} & \leq \Sigma_{\mathrm{n}=0}^{\infty} \Sigma_{\mathrm{k}=\mathrm{n}+1}^{\infty} \mathrm{b}_{\mathrm{nk}} \Sigma_{\mathrm{r}=\mathrm{n}}^{\mathrm{k}-1}\left|\Delta \mathrm{x}_{\mathrm{r}}\right| \\
& =\Sigma_{r=0}^{\infty}\left|\Delta \mathrm{x}_{\mathrm{r}}\right| \Sigma_{\mathrm{n}=0}^{\mathrm{r}} \Sigma_{\mathrm{k}=\mathrm{r}+1}^{\infty} \mathrm{b}_{\mathrm{nk}} \\
& =\Sigma_{r=0}^{\infty}\left|\Delta \mathrm{x}_{\mathrm{r}}\right| \mathrm{D}_{\mathrm{r}}, \text { say. }
\end{aligned}
$$

By the Lemma following, $C_{r}=O(\sqrt{r})$ and $D_{r}=O(\sqrt{r})$, so

$$
C+D \leq H \Sigma_{r=0}^{\infty}\left|\Delta x_{r}\right| \sqrt{r}<\infty,
$$

which proves the theorem.

LEMMA. If $b_{n k}=e^{-n k} n^{k} / k$ ! and $r$ is a positive integer, then

$$
\sum_{n=r+1}^{\infty} \Sigma_{k=0}^{r} b_{n k}=0(\sqrt{r})
$$

and

$$
\sum_{\mathrm{n}=0^{\mathrm{r}}}^{\mathrm{r}} \sum_{\mathrm{k}=\mathrm{r}+1}^{\infty} \mathrm{b}_{\mathrm{nk}}=0(\sqrt{\mathrm{r}}) \text {. }
$$

PROOF. Let $p=[\sqrt{r}]$, and write the sum in (i) as

$$
\sum_{n=r+1}^{\infty} \Sigma_{k=0}^{r-p} b_{n k}+\Sigma_{n=r+1}^{\infty} \Sigma_{k=r-p+1}^{r} b_{n k}=F_{r}+G_{r} \text {, say. }
$$

If $s<n$, then (cf. [2, p. 202]) 


$$
\begin{aligned}
\sum_{k=0}^{s} \frac{n^{k}}{k !} & =\frac{n^{s}}{s !}\left(1+\frac{s}{n}+\frac{s}{n} \frac{s-1}{n}+\cdots\right) \\
& \leq \frac{n^{s}}{s !}\left(1+\frac{s}{n}+\left(\frac{s}{n}\right)^{2}+\cdots\right) \\
& =\frac{n s}{s !}\left(\frac{n}{n-s}\right) .
\end{aligned}
$$

In $F_{r}$ we have $s=r-p$ and

$$
\max _{n \geq r+1} \frac{n}{n-r+p}=\frac{r+1}{p+1} \leq \sqrt{r}+1,
$$

so

$$
F_{r}<(\sqrt{r}+1) \frac{1}{(r-p) !} \sum_{n=r+1}^{\infty} e^{-n_{n} r-p} \leq \sqrt{r}+1 .
$$

In $G_{r}$ we have

$$
\Sigma_{k=r-p+1}^{r} b_{n k} \leq \sqrt{r} \max _{k<r} b_{n k}=\sqrt{r} e^{-n} \frac{n^{r}}{r !},
$$

so

$$
G_{r} \leq \sqrt{r} \frac{1}{r !} \Sigma_{n=r+1}^{\infty} e^{-n n} \leq \sqrt{r} .
$$

Hence, (i) is proved.

Next write the sum in (ii) as

$$
\Sigma_{n=0}^{r} \Sigma_{k=r+1}^{r+p-1} b_{n k}+\Sigma_{n=0}^{r} \Sigma_{k=r+p}^{r} b_{n k}=H_{r}+I_{r} \text {, say. }
$$

(Assume that $\mathrm{H}_{\mathrm{r}}=0$ if $\mathrm{p}=1$. ) Then

$$
\begin{aligned}
H_{r} & \leq(p-1) \sum_{n=0}^{r} e^{-n} \max _{k>r} \frac{n^{k}}{k !} \\
& \leq(\sqrt{r}-1) \frac{1}{(r+1) !} \sum_{n=0}^{x} e^{-n_{n} r+1} \\
& \leq \sqrt{r}-1 .
\end{aligned}
$$

If $s \geq n$, then

$$
\begin{aligned}
\sum_{k=s}^{\infty} \frac{n^{k}}{k !} & =\frac{n^{s}}{s !}\left(1+\frac{n}{s+1}+\frac{n}{s+1} \frac{n}{s+2}+\cdots\right) \\
& \leq \frac{n^{s}}{s !}\left(1+\frac{n}{s+1}+\left(\frac{n}{s+1}\right)^{2}+\cdots\right)
\end{aligned}
$$




$$
=\frac{n^{s}}{s !}\left(\frac{s+1}{s+1-n}\right) \text {. }
$$

Taking $s=r+p$, we have

$$
\begin{aligned}
I_{r} & \leq \frac{1}{(r+p) !} \sum_{n=0}^{r} e^{-n_{n} r+p}\left(\frac{r+p+1}{r+p+1-n}\right) \\
& \leq\left(\frac{r+p+1}{p+1}\right) \frac{1}{(r+p) !} \sum_{n=0}^{r} e^{-n_{n} r+p} \\
& \leq \sqrt{r}+1
\end{aligned}
$$

This completes the proof of the Lemma.

By combining Theorem 3 with Theorem 1 , we get an $l-l$ Tauberian theorem for the Euler-Knopp means.

THEOREM 4. If $r>0$ and $x$ is a sequence satisfying (*) such that $E_{r} x$ is in $\ell$, then $x$ is in $\ell$.

Next we give an application of these Tauberian theorems.

EXAMPLE. The following sequence is not mapped into $l$ by $B$-- or, a fortiori, by $\mathrm{E}_{\mathrm{r}}$, with $\mathrm{r}>0$. Define $\mathrm{x}$ by

$$
x_{0}=\pi^{2} / 6 \text { and } \Delta x_{j}=1 /(j+1)^{2} \text {. }
$$

Then $x$ satisfies (*), but $x$ is not in $\ell$ because if $k \geq 1$,

$$
\begin{aligned}
x_{k} & =x_{0}-\sum_{j=0}^{k-1} \Delta x_{j}=\frac{\pi^{2}}{6}-\sum_{m=1}^{k} m^{-2} \\
& =\Sigma_{m \geq k+1} m^{-2} \sim 1 / k .
\end{aligned}
$$

Hence, by Theorem $3, \mathrm{Bx}$ is not in $\ell$.

It is possible -- but much more tedious -- to construct a real number sequence $x$ such that $\Delta x_{j}= \pm(j+1)^{-2}$ and $x$ changes sign infinitely many times, yet $x$ is not in $\ell$. For such an $x$, Theorem 3 implies that Bx cannot be in $l$. 


\section{REFERENCES}

1. Fridy, J. A., Absolute summability matrices that are stronger than the identity mapping, Proc. Amer. Math. Soc. 47(1975) 112-118.

2. Hardy, G. H., Divergent Series, Clarendon Press, Oxford, 1949.

3. Hardy, G. H. and J. E. Littlewood, Theorems concerning the summability of series by Borel's exponential method, Rendiconti Palermo, 41(1916) 36-53.

4. Hardy, G. H. and J. E. Littlewood, On the Tauberian theorem for Borel summability, J. London Math. Soc. 18 (1943) 194-200.

5. Knopp, K. and G. G. Lorentz, Beitrage zur absoluten Limitierung, Arch. Math. 2 (1949) 10-16.

6. Powe11, R. E. and S. M. Shah, Summability Theory and its Applications, Van Nostrand Reinhold, London, 1972. 


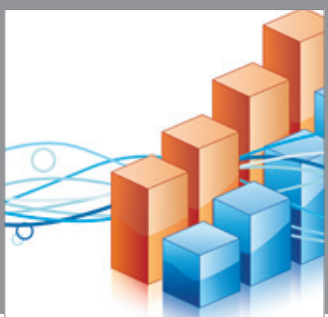

Advances in

Operations Research

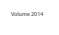

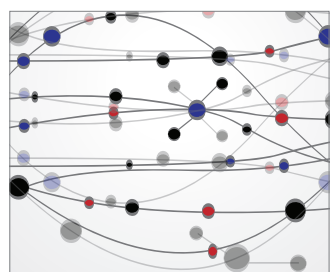

\section{The Scientific} World Journal
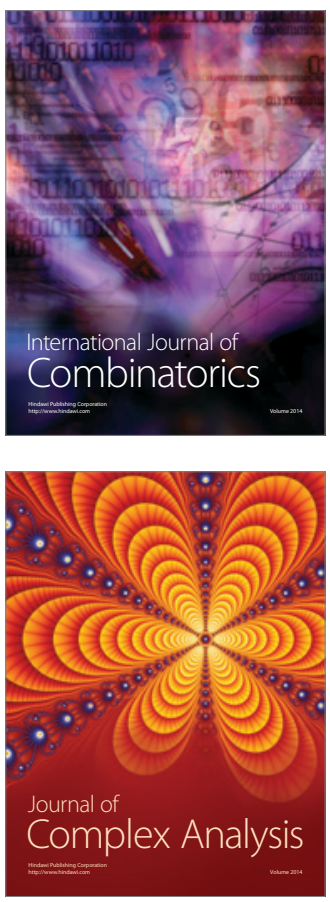

International Journal of

Mathematics and

Mathematical

Sciences
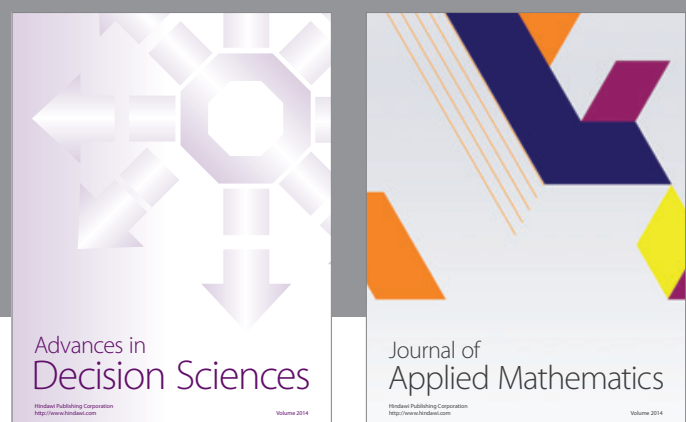

Journal of

Applied Mathematics
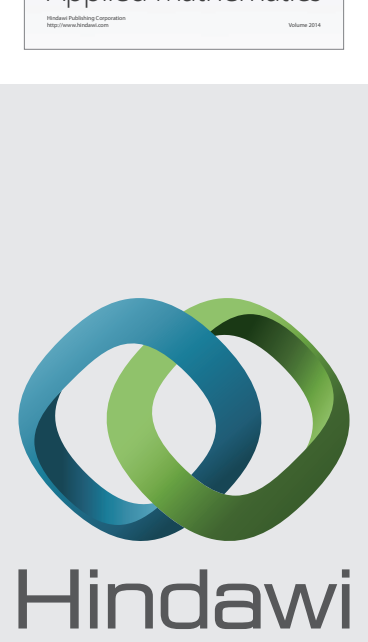

Submit your manuscripts at http://www.hindawi.com
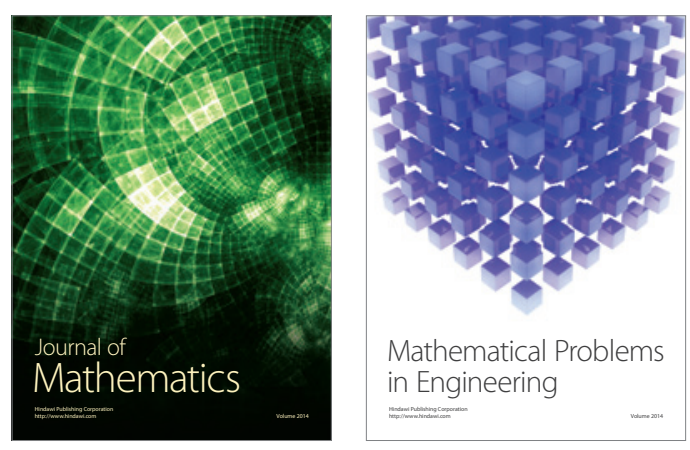

Mathematical Problems in Engineering
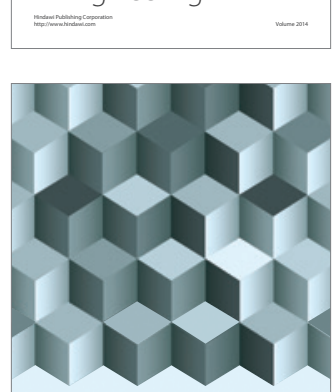

Journal of

Function Spaces
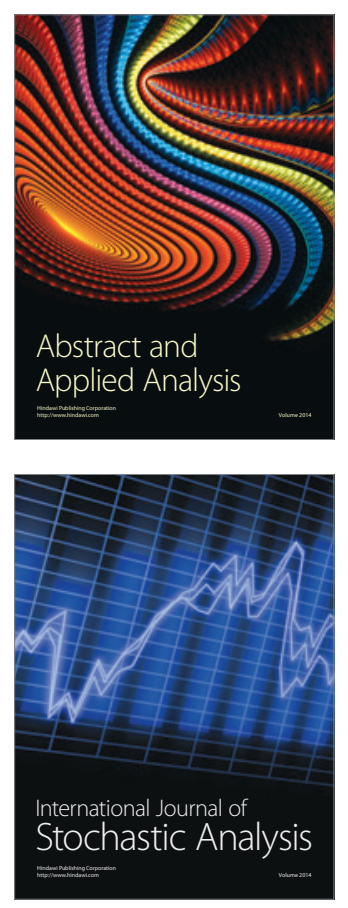

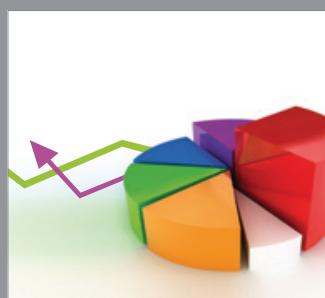

ournal of

Probability and Statistics

Promensencen
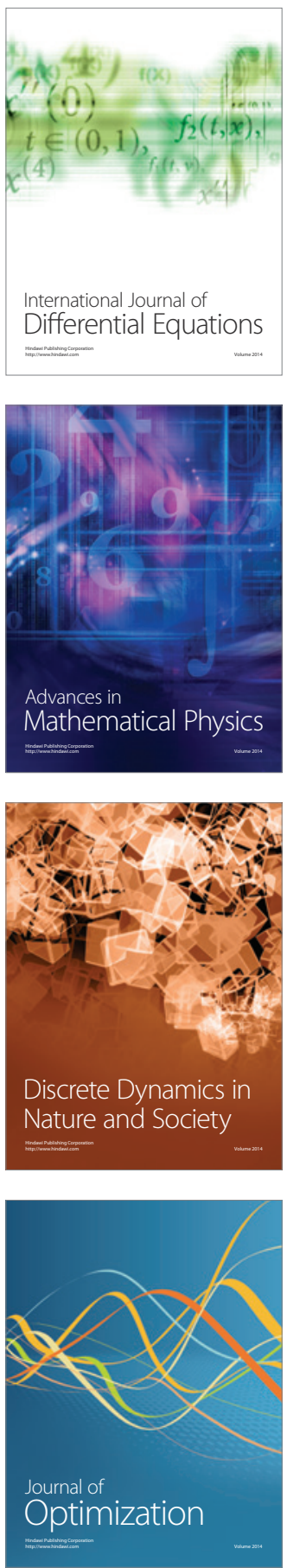